Article

\title{
Exact Solutions to Time-Fractional Fifth Order KdV Equation by Trial Equation Method Based on Symmetry
}

\section{Tao Liu}

College of Information Science and Engineering, China University of Petroleum-Beijing, Beijing 102249, China; liutao@cup.edu.cn

Received: 17 May 2019; Accepted: 27 May 2019; Published: 1 June 2019

\begin{abstract}
We study a fifth order time-fractional KdV equation (FKdV) under meaning of the conformal fractional derivative. By trial equation method based on symmetry, we construct the abundant exact traveling wave solutions to the FKdV equation. These solutions show rich evolution patterns including solitons, rational singular solutions, periodic and double periodic solutions and so forth. In particular, under the concrete parameters, we give the representations of all these solutions.
\end{abstract}

Keywords: trial equation method; symmetry; traveling wave solutions; conformal fractional derivative

\section{Introduction}

Fractional calculus has been become a hot field and applied to physics, mechanics, chemistry, biology, automatic control and so forth [1-6]. For classical Riemann-Liouville type fractional calculus, however, the properties of derivatives are more complicated than the usual derivative. For example, the Leibnitz rule does not hold. In fact, Tarasov had proved some important theorems in theory to point out that Leibnitz rule in general is invalid for Riemann-Liouville type of fractional derivative [7]. On the other hand, there are some serious mistakes in existed theory such as Jumarie's fractional derivative. Recently, Liu provided an important development in the field since he gave counterexamples to shown that Jumarie's basic formulas are not right $[8,9]$. Respectively, all those results based on the Jumarie's formulas are also wrong. Furthermore, Liu [10] proved strictly that the local fractional derivative did not exist at all on any interval. These important results tell us that we should carefully verify the basic formulas when we use the fractional derivative. To overcome the weaknesses of usual fractional derivative, a conformal fractional derivative is introduced, which satisfies routine rules of derivative, and hence draws attentions of a lot of scientists. In particular, the conformable derivative provide a tool to describes some power law behaviors in local. Therefore, it is a meaningful topic to study solutions of conformable fractional differential equations.

For convenience, we list in the following the definition and basic properties of conformal fractional derivative. For a given function $f=f(t)$ which is defined on an interval $(0,+\infty)$, and a fixed $\alpha \in(0,1)$, we define the conformal fractional derivative [11].

$$
D_{t}^{\alpha}(f(t))=\lim _{h \rightarrow 0} \frac{f\left(t+h t^{1-\alpha}\right)-f(t)}{h} .
$$

If the above limit exists, the function $f(t)$ is called $\alpha$-derivatible at $t$. The basic properties of the conformal fractional derivative can be found in [11,12]. Here we need the following formulas

(i) $D_{t}^{\alpha}\left(f(g(t))=t^{1-\alpha} g^{\alpha-1} g^{\prime}(t) D_{g}^{\alpha} f(g)=t^{1-\alpha} g^{\prime}(t) f^{\prime}(g)\right.$;

(ii) $D_{t}^{\alpha} f(t)=t^{1-\alpha} f^{\prime}(t)$. 
We can find the detailed proofs in [11,12], and other related studies and applications can be found in [13-18].

In the paper, we study a conformal time-fractional fifth order KdV equation. The usual KdV equation is the most famous integrable model, and the exact solutions have been studied extensively for integer and fractional order cases [19-28]. However, in [26,27], because the authors use the Jumarie's formulas, the obtained results are wrong. Since there is a variable index $\alpha$, the fractional derivative model has better adaptability. For example, if the mass is variable of time by power law, inertial force $m(t) u^{\prime \prime}(t)$ will be represented in terms of conformable derivative. Finding physical meaning and applications of conformable derivative is still a valuable topic. At the same time, because the order of derivative of the equation is five, we cannot directly change it into an integral form. Therefore, we use a powerful mathematical tool namely trial equation method to find an integrable factor of nonlinear fifth order KdV differential operator. The trial equation method is proposed by Liu in 2005, and then has been developed and applied to many nonlinear problems [29-32]. Comparing with other direct expansion methods, Liu's method is based on a deep idea in mathematics, that is, factorization of nonlinear differential operators. In practice, we often combine the trial equation method with the complete discrimination system for polynomial [33-38]. By using Liu's method, we need not assume the forms of solutions, while we can directly derive out all solutions of the integral and give the complete classification of these solutions according to the parameters. Up to now, these two methods have become the routine tools of giving the exact solutions to nonlinear partial differential equations. In the paper, based on symmetry, we reduce the time-fractional fifth order KdV equation to a less order equation, and we use Liu's methods to obtain its abundant exact solutions.

The paper is organized as follows. In Section 2, we give the outline of trial equation method. In Section 3, by trial equation method based on symmetry. We reduce the FKdV equation to an integral form and give its exact solutions. The last section is a short conclusion.

\section{Outline of trial Equation Method}

The main idea of trial equation method is to separate an integrable sub-equation for the considered high order nonlinear ordinary differential equation (ODE). The detail on the computations of the method can be given only for concrete problem. Here we give the main steps of trial equation method. In next section, we will give the whole computations for fractional $\mathrm{KdV}$ equation.

We generalize the Liu's trial equation method based on symmetry [29-32] to time-fractional partial differential equations as follows:

Step 1. Consider the following nonlinear time-fractional partial differential equation which does not contain explicitly $x$ and $t$ :

$$
P\left(u, D_{t}^{\alpha} u, u_{x},\left(D_{t}^{\alpha}\right)^{2} u, D_{t}^{\alpha} u_{x}, u_{x x}, \cdots\right)=0 .
$$

Take fractional traveling wave transformation

$$
u(\xi)=u(x, t), \xi=x-\frac{\omega}{\alpha} t^{\alpha} .
$$

By plugging Equation (3) into Equation (2), we get an ordinary differential equation

$$
M\left(u, u^{\prime}, \cdots\right)=0 .
$$

In general, the ODE has high order derivative and hence cannot be directly transformed to the form of an integral, so we need next step if we want to find exact solutions.

Step 2. we take the trial equation

$$
\left(u^{\prime}\right)^{2}=F(u)
$$


and by the symmetry, that is, the equation is invariant under the transformations $\xi^{*}=\xi+c$ and $u^{*}=u$, the order of the equation on $F$ can be reduced one. Therefore we let $F(u)=\sum_{i=0}^{m} a_{i} u^{i}$, where $m$ and $a_{i}(i=0, \cdots, m)$ are unknown constants which will be determined. By plugging Equation (5) and other derivative terms into Equation (4), we get a polynomial $G(u)$. By using the balance principle we can solve the value of $m$. By taking the coefficients of $G(u)$ to be zeroes, we obtain an algebraic equations system from which we give the values of $a_{0}, \cdots, a_{m}$.

Step 3. we give another form of the Equation (5)

$$
\pm\left(\xi-\xi_{0}\right)=\int \frac{1}{\sqrt{F(u)}} \mathrm{d} u .
$$

Now by the complete discrimination system of polynomial $F(u)$ [28-30], we can classify the solutions of Equation (6) from which we get abundant exact solutions to Equation (1).

Remark. For some differential equations, in the trial Equation (5), $F(u)$ is perhaps a more general functions rather than a polynomial. There exist no general principle to give the form of trial equation, so we need a trial procedure to choose it according to the structure of the considered problem. We can find more details of the trial equation method in a series of Liu's papers [29-33].

\section{Exact Solutions of FKdV Equation}

We consider the following fifth order FKdV equation

$$
D_{t}^{\alpha} u+a u^{2} u_{x}+b u_{3 x}+c u_{5 x}=0,
$$

where $a, b, c$ are model parameters. When we consider variable mass with respect to time by power law, the usual time-derivative in corresponding $\mathrm{KdV}$ equation can be replaced by conformable derivative, and hence we will get the Equation (7). Substituting fractional traveling wave transformation (3) into Equation (7) and integrating it once yield the equation,

$$
c_{0}-\omega u+\frac{a}{3} u^{3}+b u^{\prime \prime}+c u^{(4)}=0
$$

Substituting trial Equation (5) into Equation (8), we get

$$
\frac{c}{2} F F^{\prime \prime \prime}+\frac{c}{4} F^{\prime} F^{\prime \prime}+\frac{b}{2} F^{\prime}+c_{0}-\omega u+\frac{a}{3} u^{3}=0 .
$$

Then we take $F(u)=\sum_{i=0}^{m} a_{i} u^{i}$, and according to the balance principle we know $m=3$. Therefore, the trial equation is taken as

$$
\left(u^{\prime}\right)^{2}=a_{3} u^{3}+a_{2} u^{2}+a_{1} u+a_{0} .
$$

Substituting the trial equation into the Equation (9) to get the algebraic equations system

$$
\begin{gathered}
\frac{a}{3}+\frac{15 c}{2} a_{3}^{3}=0, \quad 3 b+5 c a_{2}=0 \\
\omega+b a_{2}+6 c a_{1} a_{3}+c a_{0}^{2}=0, \\
c_{0}+\frac{a_{1} b}{2}+3 c a_{0} a_{3}+c a_{1} a_{2}=0 .
\end{gathered}
$$

Solving above algebraic equations system, we obtain 


$$
\begin{gathered}
a_{3}= \pm \sqrt{-\frac{2 a}{45 c}}, a_{2}=-\frac{3 b}{5 c} \\
\omega=b a_{2}+6 c a_{1} a_{3}+c a_{0}^{2}, \\
c_{0}=-\frac{a_{1} b}{2}-3 c a_{0} a_{3}-c a_{1} a_{2},
\end{gathered}
$$

where $a_{0}$ and $a_{1}$ can be taken as arbitrary constants. Under the condition (12), we give exact solutions to fractional KdV equation from the solutions of Equation (10). Without loss of generalization, we assume $a_{3}>0$, otherwise, we replace $u$ by $-u$. Then we have

$$
\pm \sqrt{a_{3}}\left(\xi-\xi_{0}\right)=\int \frac{1}{\sqrt{u^{3}+d_{2} u^{2}+d_{1} u+d_{0}}} \mathrm{~d} u
$$

where $d_{2}=\frac{a_{2}}{a_{3}}, d_{1}=\frac{a_{1}}{a_{3}}, d_{0}=\frac{a_{0}}{a_{3}}$. Denote

$$
\begin{gathered}
F(u)=u^{3}+d_{2} u^{2}+d_{1} u+d_{0}, \\
\triangle=-27\left(\frac{2 d_{2}^{3}}{27}+d_{0}-\frac{d_{1} d_{2}}{3}\right)^{2}-4\left(d_{1}-\frac{d_{2}^{2}}{3}\right)^{3}, \\
D_{1}=d_{1}-\frac{d_{2}^{2}}{3} .
\end{gathered}
$$

Then the complete discrimination system for $F(u)$ is made up by $\triangle$ and $D_{1}$. According to the cases of roots, we have the following four cases [26].

Family 1. $\triangle=0, D_{1}<0$. Then $F(u)=\left(u-\beta_{1}\right)^{2}\left(u-\beta_{2}\right), \beta_{1} \neq \beta_{2}$. Three solutions are given by

$$
\begin{gathered}
u_{1}=\left(\beta_{1}-\beta_{2}\right) \tanh ^{2}\left(\frac{\sqrt{\beta_{1}-\beta_{2}}}{2} \sqrt{a_{3}}\left(x-\frac{\omega}{\alpha} t^{\alpha}-\xi_{0}\right)\right)+\beta_{2}, \\
u_{2}=\left(\beta_{1}-\beta_{2}\right) \operatorname{coth}^{2}\left(\frac{\sqrt{\beta_{1}-\beta_{2}}}{2} \sqrt{a_{3}}\left(x-\frac{\omega}{\alpha} t^{\alpha}-\xi_{0}\right)\right)+\beta_{2}, \beta_{1}>\beta_{2} . \\
u_{3}=-\beta_{1}+\beta_{2} \tan ^{2}\left(\frac{\sqrt{-\beta_{1}+\beta_{2}}}{2} \sqrt{a_{3}}\left(x-\frac{\omega}{\alpha} t^{\alpha}-\xi_{0}\right)\right)+\beta_{2}, \beta_{1}<\beta_{2} .
\end{gathered}
$$

Next under concrete parameters we give the realizations of these solutions. Take $a_{0}=a_{3}$, $a_{1}=a_{2}=-a_{3}$, then $\beta_{1}=1, \beta_{2}=-1$. The corresponding solutions are given by

$$
\begin{aligned}
& u_{1}=2 \tanh ^{2}\left(\frac{\sqrt{2}}{2} \sqrt{a_{3}}\left(x-\frac{\omega}{\alpha} t^{\alpha}-\xi_{0}\right)\right)-1, \\
& u_{2}=2 \operatorname{coth}^{2}\left(\frac{\sqrt{2}}{2} \sqrt{a_{3}}\left(x-\frac{\omega}{\alpha} t^{\alpha}-\xi_{0}\right)\right)-1 .
\end{aligned}
$$

Take $a_{0}=a_{1}=-a_{3}, a_{2}=a_{3}$, then $\beta_{1}=-1, \beta_{2}=1$. The corresponding solution is given by

$$
u_{3}=2 \tan ^{2}\left(\frac{\sqrt{2}}{2} \sqrt{a_{3}}\left(x-\frac{\omega}{\alpha} t^{\alpha}-\xi_{0}\right)\right)+1 .
$$

Family 2. $\triangle=0, D_{1}=0$. Then $F(u)=(u-\beta)^{3}$. We obtain

$$
u_{4}=4 \sqrt{a_{3}}\left(x-\frac{\omega}{\alpha} t^{\alpha}-\xi_{0}\right)^{-2}+\beta .
$$

Take $a_{0}=-a_{3}, a_{1}=3 a_{3}, a_{2}=-2 a_{3}$, then $\beta=1$. The corresponding solution is given by

$$
u_{4}=4 \sqrt{a_{3}}\left(x-\frac{\omega}{\alpha} t^{\alpha}-\xi_{0}\right)^{-2}+1 .
$$


Family 3. $\triangle>0, D_{1}<0$. We have $F(u)=\left(u-\beta_{1}\right)\left(u-\beta_{2}\right)\left(u-\beta_{3}\right)$. Then we give two solutions

$$
\left.u_{5}=\beta_{1}+\left(\beta_{2}-\beta_{1}\right)^{2}\left(\frac{\sqrt{\beta_{3}-\beta_{1}}}{2} \sqrt{a_{3}}\left(x-\frac{\omega}{\alpha} t^{\alpha}-\xi_{0}\right)\right), m\right),
$$

and

$$
u_{6}=\frac{\left.\beta_{3}-\beta_{2}^{2}\left(\frac{\sqrt{\beta_{3}-\beta_{1}}}{2} \sqrt{a_{3}}\left(x-\frac{\omega}{\alpha} t^{\alpha}-\xi_{0}\right)\right), m\right)}{\left.2\left(\frac{\sqrt{\beta_{3}-\beta_{1}}}{2} \sqrt{a_{3}}\left(x-\frac{\omega}{\alpha} t^{\alpha}-\xi_{0}\right)\right), m\right)},
$$

where $m^{2}=\frac{\beta_{2}-\beta_{1}}{\beta_{3}-\beta_{1}}$.

Take $a_{0}=-2 a_{3}, a_{1}=-a_{3}, a_{2}=2 a_{3}$, then $\beta_{1}=-2, \beta_{2}=-1, \beta_{3}=1$. The corresponding solutions are given by

$$
u_{5}=-2+^{2}\left(\frac{\sqrt{3 a_{3}}}{2}\left(x-\frac{\omega}{\alpha} t^{\alpha}-\xi_{0}\right), \frac{1}{\sqrt{3}}\right) .
$$

for $-2<u<-1 w>1$, and

$$
u_{6}=\frac{\left.1+1^{2}\left(\frac{\sqrt{3 a_{3}}}{2}\left(x-\frac{\omega}{\alpha} t^{\alpha}-\xi_{0}\right)\right), \frac{1}{\sqrt{3}}\right)}{\left.2\left(\frac{\sqrt{3 a_{3}}}{2}\left(x-\frac{\omega}{\alpha} t^{\alpha}-\xi_{0}\right)\right), \frac{1}{\sqrt{3}}\right)},
$$

for $u>1$.

Family 4. $\triangle<0$. Now we get $F(u)=(u-\beta)\left(u^{2}+p u+q\right)$ where we also have $p^{2}-4 q<0$. Furthermore, we get

$$
u_{7}=\beta-\sqrt{\beta^{2}+p \beta+q}+\frac{2 \sqrt{\beta^{2}+p \beta+q}}{1+\left(\left(\beta^{2}+p \beta+q\right)^{\frac{1}{4}} \sqrt{a_{3}}\left(x-\frac{\omega}{\alpha} t^{\alpha}-\xi_{0}\right), m\right)},
$$

where $m^{2}=\frac{1}{2}\left(1-\frac{\beta+\frac{p}{2}}{\sqrt{\beta^{2}+p \beta+q}}\right)$.

Take $a_{0}=-2 a_{3}, a_{1}=-a_{3}, a_{2}=-a_{3}$, then $\beta=2, p=1, q=1$. The corresponding solution is given by

$$
u_{7}=2-\sqrt{7}+\frac{2 \sqrt{7}}{1+\left(7^{\frac{1}{4}} \sqrt{a_{3}}\left(x-\frac{\omega}{\alpha} t^{\alpha}-\xi_{0}\right), \frac{\sqrt{2-\sqrt{7}}}{2}\right)} .
$$

From the above discussions, we obtain abundant exact solutions for the fifth order FKdV equation, in which $u_{1}$ and $u_{2}$ are two solutions, $u_{3}$ is a periodic solution, $u_{4}$ is singular rational solution, $u_{5}, u_{6}$ and $u_{7}$ are double periodic elliptic function solutions. In particular, for the concrete parameters, we give these concrete solutions. These solutions show abundant evolution patterns. We also point out that when $\alpha=1$, these solutions give solutions for the integer order $\mathrm{KdV}$ equation.

\section{Conclusions and Discussions}

Fractional fifth order $\mathrm{KdV}$ equation is a typical high order rank homogenous partial differential equation, which in general cannot be directly transformed into the integral form. It is just by trial equation method so that we reduce it to an integrable first order equation from which four kinds of exact solutions are found. These solutions show rich traveling wave patterns.

In general, to find exact solutions for nonlinear ODES and PDEs is a very difficult problem. The Liu's trial equation method provides a useful tool to do so by finding an integrable factor of the nonlinear operator. A large number of applications have shown the power of the method. Our results give a further example for such application in fractional differential equations. It is no doubt that the trial equation method will be applied to more conformable fractional order problems. 
For other more high order equations, such as generalized seventh order KdV equation

$$
D_{t}^{\alpha} u+a u^{k} u_{x}+b u_{3 x}+c u_{5 x}+d u_{7 x}=0,
$$

the trial equation method can also be applied. But we need to take a rational trial equation

$$
\left(u^{\prime}\right)^{2}=\frac{F(u)}{G(u)}
$$

where $F(u)$ is a $(k+6)$-th order polynomial, and $G(u)$ is of third order, if $\frac{k}{3}$ is not an integer. If $\frac{k}{3}$ is an integer, then the trial equation can be taken as

$$
\left(u^{\prime}\right)^{2}=F(u)
$$

where $F(u)$ is a $\left(\frac{k}{3}+2\right)$-th order polynomial. For example, we take $k=3$, then $F(u)$ is of third order. Of course, sometimes $F(u)$ will be an irrational function. In summary, the trial equation method can be used to effectively find the exact solutions to fractional conformal partial differential equations.

In final, we point out that some interesting physical problems on fractal medium $[39,40]$ which show scale properties are maybe related to the conformable fractional derivative. Indeed, finding applications of such fractional derivatives for physical phenomenon is still a meaningful topic.

Author Contributions: T.L. contributed to the conception of the study and drafted the manuscript.

Funding: This research was funded by Science Foundation of China University of Petroleum-Beijing (No. 2462019BJRC003) and the APC was also funded by Science Foundation of China University of Petroleum-Beijing (No. 2462019BJRC003).

Conflicts of Interest: The authors declare no conflict of interest.

\section{References}

1. Tarasov, V.E. Fractional Dynamics: Applications of Fractional Calculus to Dynamics of Particles, Fields and Media; Springer Science \& Business Media: Beijing, China, 2011.

2. Das, S. Functional Fractional Calculus; Springer Science Business Media: Berlin/Heidelberg, Germany, 2011.

3. El-Nabulsi, R.A. Path integral formulation of fractionally perturbed Lagrangian oscillators on fractal. J. Stat. Phys. 2018, 172, 1617-1640. [CrossRef]

4. Dong, J.; $\mathrm{Xu}, \mathrm{M}$. Space-time fractional Schrodinger equation with time-independent potentials. J. Math. Anal. Appl. 2008, 344, 1005-1017. [CrossRef]

5. Bayın, S.Ş. On the consistency of the solutions of the space fractional Schrodinger equation. J. Math. Phys. 2012, 53, 042105. [CrossRef]

6. El-Nabulsi, R.A. Emergence of quasiperiodic quantum wave functions in Hausdorff dimensional crystals and improved intrinsic Carrier concentrations. J. Phys. Chem. Solids 2019, 127, 224-230. [CrossRef]

7. Tarasov, V.E. On chain rule for fractional derivatives. Commun. Nonlinear Sci. Numer. Simul. 2016, 30, 1-4. [CrossRef]

8. Liu, C.S. Counterexamples on Jumarie's two basic fractional calculus formulae. Commun. Nonlinear Sci. Numer. Simul. 2015, 22, 92-94. [CrossRef]

9. Liu, C.S. Counterexamples on Jumarie's three basic fractional calculus formulae for non-differentiable continuous functions. Chaos Solitons Fractals 2018, 109, 219-222. [CrossRef]

10. Liu, C.S. On the local fractional derivative of everywhere non-differentiable continuous functions on intervals. Commun. Nonlinear Sci. Numer. Simul. 2017, 42, 229-235. [CrossRef]

11. Khalil, R.; Al Horani, M.; Yousef, A.; Sababheh, M. A new definition of fractional derivative. J. Comput. Appl. Math. 2014, 264, 65-70. [CrossRef]

12. Abdeljawad, T. On conformable fractional calculus. J. Comput. Appl. Math. 2015, 279, 57-66. [CrossRef]

13. Anderson, D.R.; Ulness, D.J. Properties of the Katugampola fractional derivative with potential application in quantum mechanics. J. Math. Phys. 2015, 56, 063502. [CrossRef] 
14. Eslami, M.; Rezazadeh, H. The first integral method for Wu-Zhang system with conformable time-fractional derivative. Calcolo 2016, 53, 475-485. [CrossRef]

15. Korkmaz, A.; Hosseini, K. Exact solutions of a nonlinear conformable time-fractional parabolic equation with exponential nonlinearity using reliable methods. Opt. Quantum Electron 2017, 49, 278. [CrossRef]

16. Çenesiz, Y.; Baleanu, D.; Kurt, A.; Tasbozan, O. New exact solutions of Burgers's type equations with conformable derivative. Waves Random Complex Media 2017, 27, 103-116. [CrossRef]

17. Zhao, D.; Luo, M. General conformable fractional derivative and its physical interpretation. Calcolo 2017, 54, 903-917. [CrossRef]

18. Caponetto, R.; Dongola, G.; Fortuna, L.; Gallo, A. New results on the synthesis of FO-PID controllers. Commun. Nonlinear Sci. Numer. Simul. 2010, 15, 997-1007. [CrossRef]

19. Abbasbandy, S.; Zakaria, F.S. Soliton solutions for the fifth-order KdV equation with the homotopy analysis method. Nonlinear Dyn. 2008, 51, 83-87. [CrossRef]

20. Wazwaz, A.M. The extended tanh method for new solitons solutions for many forms of the fifth-order KdV equations. Appl. Math. Comput. 2007, 184, 1002-1014. [CrossRef]

21. Kaya, D.; El-Sayed, S.M. On a generalized fifth order KdV equations. Phys. Lett. A 2003, 310, 44-51. [CrossRef]

22. Chun, C. Solitons and periodic solutions for the fifth-order KdV equation with the Exp-function method. Phys. Lett. A 2008, 372, 2760-2766. [CrossRef]

23. Parkes, E.J. Exact solutions to the two-dimensional Korteweg-de Vries-Burgers equation. Phys. Lett. A 1994, 27, 497-502. [CrossRef]

24. Cooper, F.; Hyman, L.M.; hare, A.K. Compacton solutions in a class of generalized fifth-order Korteweg-de Vries equations. Phys. Rev. E 2001, 64, 026608. [CrossRef] [PubMed]

25. Wazwaz, A.M. Solitons and periodic solutions for the fifth-order KdV equation. Appl. Math. Lett. 2006, 19, 1162-1167. [CrossRef]

26. Lu, D.; Yue, C.; Arshad, M. Traveling Wave Solutions of Space-Time Fractional Generalized Fifth-Order KdV Equation. Adv. Math. Phys. 2017, 2017. [CrossRef]

27. Wang, G.W.; Xu, T.Z.; Feng, T. Lie Symmetry Analysis and Explicit Solutions of the Time Fractional Fifth-Order KdV Equation. PLoS ONE 2014, 9, e88336. [CrossRef]

28. Liu, H. Complete Group Classifications and Symmetry Reductions of the Fractional Fifth-Order KdV Types of Equations. Stud. Appl. Math. 2013, 131, 317-330. [CrossRef]

29. Liu, C.S. Trial equation emthod and its applications to nolinear evolution equations. Acta Phys. Sin. 2005, 54, 2505-2509.

30. Liu, C.S. A new trial equation method and its applications. Commun. Theor. Phys. 2006, 45, 395-397.

31. Liu, C.S. Trial equation method for nonlinear evolution equations with rank inhomogeneous: mathematical discussions and applications. Commun. Theor. Phys. 2006, 45, 219-223.

32. Liu, C.S. Using trial equation method to solve the exact solutions for two kinds of KdV equations with variable coefficients. Acta. Phys. Sin. 2005, 54, 4506-4510.

33. Liu, C.S. Trial equation method based on symmetry and applications to nonlinear equations arising in mathematical physics. Found. Phys. 2011, 41, 793-804. [CrossRef]

34. Liu, C.S. Applications of complete discrimination system for polynomial for classifications of traveling wave solutions to nonlinear differential equations. Comput. Phys. Commun. 2010, 181, 317-324. [CrossRef]

35. Cheng-shi, L. Traveling wave solutions of a kind of generalized Ginzburg-Landau equation. Commun. Theor. Phys. 2005, 43, 787-790. [CrossRef]

36. Cheng-shi, L. Travelling wave solutions of triple Sine-Gordon equation. Chin. Phys. Lett. 2004, 21, $2369-2371$. [CrossRef]

37. Cheng-shi, L. New exact envelope traveling wave solutions to higher-order dispersive cubic-qintic nonlinear Schrödinger equation. Commun. Theor. Phys. 2005, 44, 799-801.

38. Cheng-shi, L. Solution of ODE $u^{\prime \prime}+p(u)\left(u^{\prime}\right)^{2}+q(u)=0$ and applications to classifications of all single travelling wave solutions to some nonlinear mathematical physics equations. Commun. Theor. Phys. 2008, 49, 291-296. [CrossRef] 
39. Gladkov, S.O.; Bogdanova, S.B. On the question of the magnetic susceptibility of fractal ferromagnetic wires. Russ. Phys. J. 2014, 57, 469-473. [CrossRef]

40. Gladkov, S.O.; Bogdanova, S.B.; Vestyak, A.V. On physical applications of one-and two-dimensional topoloigical objects with fractal dimension. Canad. J. Phys. 2008, 96, 249-254. [CrossRef]

(C) 2019 by the authors. Licensee MDPI, Basel, Switzerland. This article is an open access article distributed under the terms and conditions of the Creative Commons Attribution (CC BY) license (http://creativecommons.org/licenses/by/4.0/). 